\title{
Evaluación del programa de detección precoz de hipoacusia en recién nacidos prematuros extremos (RNPE), experiencia Hospital Padre Hurtado
}

\author{
Hearing screening in infants with very low birth weight (VLBW), \\ Padre Hurtado experience
}

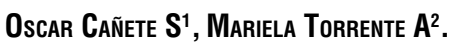

\begin{abstract}
RESUMEN
Introducción: En julio de 2005 se implementó en Chile un programa de pesquisa precoz de hipoacusia en recién nacidos menores de 32 semanas de edad gestacional y/ o con peso de nacimiento menor a 1.500 grs.

Objetivo: Evaluar la ejecución de este programa en el Hospital Padre Hurtado, durante el período julio 2005 y diciembre 2009.

Material y método: Se utilizaron potenciales auditivos evocados de tronco automatizado (PEATa) en dos etapas (pesquisa). Los pacientes que fallaron fueron estudiados mediante potenciales evocados auditivos extendidos (PEAe). Para el diagnóstico de hipoacusia sensorioneural (HSN) se consideró dos PEAe (click) alterados y audiometría de refuerzo visual concordante.

Resultados: En el período se evaluaron 166 RNPE. La tasa de referencia fue de 7,9\% (13 pacientes), considerando las fallas uni y bilaterales en dos PEATa. La tasa de HSN de esta población correspondió a 1,8\%.

Conclusiones: La tasa de referencia (7,9\%) así como de prevalencia de HSN de la población estudiada (1,8\%) está dentro de lo descrito en la literatura para recién nacidos de alto riesgo. Se sugiere una etapa de pesquisa en 2 fases, estudio de los pacientes que refieren tanto bilateralmente como unilateralmente y el seguimiento audiológico de los pacientes previamente diagnosticados.

Palabras clave: Hipoacusia del prematuro, prematuro, prematuro de bajo peso, pesquisa auditiva en el recién nacido, potenciales evocados auditivos automatizados.
\end{abstract}

\begin{abstract}
Introduction: In July 2005, Chile implemented a hearing screening program in newborns under 32 weeks gestational age and/or birth weight less than $1500 \mathrm{~g}$.

Aim: Evaluate the implementation of this program in the Hospital Padre Hurtado, during the period July 2005 and December 2009.

Material and method: We used automated auditory brainstem response (AABR) in two stages (screening). Patients who failed were studied using click auditory brainstem
\end{abstract}

\footnotetext{
${ }^{1}$ Tecnólogo Médico, Departamento de Audición y Lenguaje, Servicio de Otorrinolaringología, Hospital Padre Hurtado. Santiago, Chile ${ }^{2}$ Médico, Servicio de Otorrinolaringología, Hospital Padre Hurtado. Santiago, Chile.
} 
response (ABR). For the diagnosis of sensorineural hearing loss (SNHL) are considered two $A B R$ altered and consistent visual reinforcement audiometry.

Results: A total of 166 were evaluated VLBW. The reference rate was $7.9 \%$ (13 patients), whereas failures in two uni-and bilateral $A A B R$. SNHL rate of this population accounted for $1.8 \%$.

Conclusions: The reference rate (7.9\%) and HSN prevalence of the study population $(1.8 \%)$ is within that described in the literature for high-risk newborns. It suggests a period of research in two phases, a study of patients referred both bilaterally and unilaterally and audiological monitoring of patients previously diagnosed.

Key words: Auditory evoked potentials, auditory brainstem responses, hearing screening in newborn, hearing loss in newborn, hearing loss in very low birth weight newborns.

\section{INTRODUCCIÓN}

Está bien establecido el efecto que una pérdida auditiva no identificada al momento de nacer provoca en el adecuado desarrollo del lenguaje y habla de un niño. Esta puede tener graves consecuencias en el desempeño académico y social del niño que la presenta ${ }^{1}$.

La hipoacusia sensorioneural (HSN) bilateral es una patología presente en 2 a 5 de cada 1.000 recién nacidos vivos. Para el niño se traduce en una severa discapacidad y para la sociedad constituye la pérdida de un individuo económicamente productivo. La detección precoz es difícil ya que sólo $50 \%$ de los pacientes tiene factores de riesgo, tales como bajo peso de nacimiento $(<1.500 \mathrm{~g})$, Apgar bajo, hiperbilirrubinemia, uso de ototóxicos, TORCH, malformaciones craneofaciales, antecedente de meningitis, antecedentes familiares de hipoacusia. Por esta razón se recomienda realizar pesquisa de hipoacusia a todos los recién nacidos vivos (pesquisa universal). En poblaciones sin programas de detección precoz la edad promedio de diagnóstico de HSN se ha estimado en 30 meses $^{2}$.

Los recién nacidos con prematurez extrema (menos de 32 semanas de edad gestacional y/o menos de 1.500 gramos de peso al nacer) (RNPE) constituyen una población de mayor riesgo para HSN, algunos estudios reportan una incidencia de pérdida auditiva sensorioneural severa a profunda en esta población que fluctúa entre $2 \%$ y $4 \%{ }^{3}$. Desde julio del año 2005 existe en Chile un programa de pesquisa precoz de hipoacusia en el RNPE. Por ley se garantiza la detección, diagnóstico y habilitación auditiva de todos los niños nacidos en el país de estas características. La etapa de pesquisa se realiza mediante potenciales auditivos evocados de tronco cerebral abreviados (PEATa) en dos etapas, y el diagnóstico mediante potenciales auditivos evocados de tronco cerebral extendidos (PEATe) con estímulo "click" y audiometría de refuerzo visual.

El objetivo del presente trabajo fue evaluar la ejecución de este programa en un hospital perteneciente al sistema público de salud.

\section{MATERIAL Y MÉTODO}

Se incluyeron los pacientes con diagnóstico de prematurez extrema nacidos en el Hospital Padre Hurtado de la ciudad de Santiago, evaluados en la Unidad de Otorrinolaringología en el período entre julio de 2005 y diciembre de 2009.

Para la etapa de pesquisa se utilizó un equipo PEATa marca GSI modelo Audioscreener ${ }^{\mathrm{MR}}$, protocolo QuickABR (intensidad de estimulación de 35 $\mathrm{dBnHL}, 37,7$ est/seg. y 2.000 promediaciones). Se aplicó la evaluación en dos fases, realizándose la primera sólo cuando el RNPE presentaba al menos 34 semanas de edad gestacional. La segunda (si era necesaria) se realizó cuando el paciente presentaba al menos 40 semanas de edad gestacional. Los resultados fueron registrados en el formulario respectivo así como se almacenaron en la base de datos Audiotrack ${ }^{\mathrm{MR}}$.

En el examen PEATa se consideró como criterio de normalidad (pasa) la aparición de respuesta (automática) a una intensidad de estimulación de $35 \mathrm{~dB} \mathrm{nHL}$. Los pacientes que fallaron dos PEATa, tanto en forma unilateral como bilateral, fueron estudiados mediante PEATe, considerándose dentro de rangos de normalidad la presencia de um- 
bral de onda $\mathrm{V}$ a $30 \mathrm{~dB} \mathrm{nHL}$ en forma bilateral. En caso de observar un PEATe alterado el paciente fue evaluado por el médico especialista y si éste lo consideraba pertinente se realizó otro PEATe según fuese el caso. Para la evaluación mediante PEATe se utilizó equipo de PEAT Vivosonic Integrity v 500 y Ampliad MK22.

Todos los pacientes que fueron diagnosticados con una HSN uni o bilateral, sin importar el grado, se les realizó una audiometría de refuerzo visual así como una evaluación del posible uso de sistemas de amplificación auditiva además de una evaluación del lenguaje y habla.

\section{RESULTADOS}

Se evaluaron 166 RNPT durante el periodo julio 2005 a diciembre 2009. No disponemos de dato confiable del número de RNPE nacidos durante el periodo de estudio, razón por la cual no podemos reportar el porcentaje de cobertura del programa. De los pacientes evaluados, 86 (52\%) correspondieron a sexo masculino y $80(48 \%)$ a sexo femenino. En relación con la edad de evaluación de PEATa la media de la primera evaluación fue de 36 semanas de edad gestacional (rango de 34 a 40 semanas). Al término de la segunda fase de la etapa de pesquisa refirieron 13 pacientes lo que correspondió a una tasa del 7,9\%, de estos casos 13 refirieron en forma bilateral y 1 unilateral (Figura 1). De los 13 pacientes referidos a la etapa diagnóstica 5 presentaron HSN bilateral, lo que equivale al 3,6\% de la población estudiada (Tabla 1), los 8 restantes presentaron respuesta a 30 dBnHL en ambos oídos por lo que se descartó la presencia de hipoacusia. Cabe destacar que dos pacientes portadores de hipoacusia bilateral (moderada y leve) fallaron la etapa de pesquisa en forma unilateral (Caso 1 y 2 ).

De los 5 pacientes confirmados con HSN bilateral, 2 presentaron HSN leve, $1 \mathrm{HSN}$ moderada y 2 HSN profunda. El diagnóstico se confirmó con 2 PEATe realizados con mínimo de 2 meses de diferencia, más una audiometría de refuerzo visual (8 meses edad corregida promedio). En el seguimiento pudimos observar a través de exámenes de control (PEAT y ARV) que uno de los pacientes con hipoacusia leve (caso 2) y el paciente con hipoacusia moderada (caso 3) normalizaron su audición. Por lo tanto, la prevalencia de HSN en la población de 166 pacientes evaluados fue de 1,8\%.

\section{DISCUSIÓN}

La detección precoz de hipoacusia sensorioneural es clave para una adecuada rehabilitación del indi-

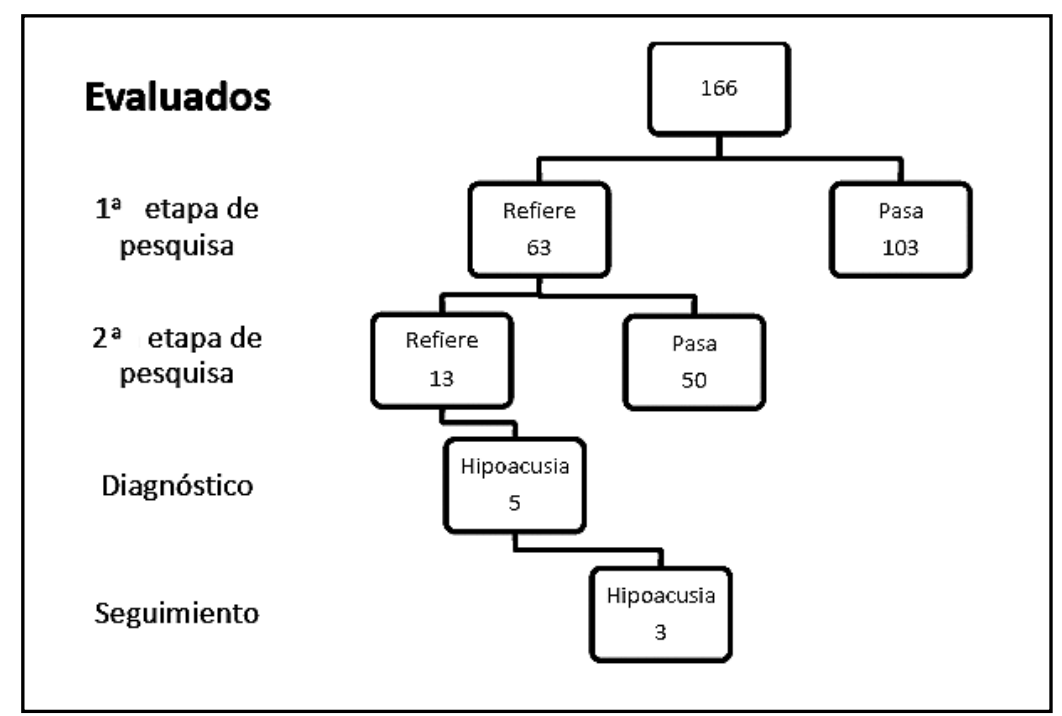

Figura 1. Resumen de pacientes evaluados programa de pesquisa de la hipoacusia en RNPE, periodo julio 2005 a diciembre 2009. 
Tabla 1. Pacientes dignosticados con hipoacusia sensorioneural bilateral

\begin{tabular}{|c|c|c|c|c|c|}
\hline Caso & $\begin{array}{l}\text { Edad gestacional } \\
\text { (semanas) }\end{array}$ & $\begin{array}{c}\text { Peso de } \\
\text { nacimiento (grs) }\end{array}$ & Apgar & $\begin{array}{l}\text { Riesgos } \\
\text { asociados }\end{array}$ & Hipoacusia \\
\hline$N^{0} 1$ & 32 & 1.490 & $3 / 6$ & $\begin{array}{c}\text { Bili 16,8 mg/dl } \\
\text { ATB }\end{array}$ & $\begin{array}{l}30 \mathrm{dBnhL} \text { bilateral } \\
\text { Sin audífono }\end{array}$ \\
\hline $\mathrm{N}^{0} 2$ & 30 & 1.560 & $9 / 9$ & $\begin{array}{c}\text { ATB } \\
\text { Bili 14,4 mg/dl } \\
\text { Audífono en OD }\end{array}$ & $\begin{array}{l}\text { OD } 50 \mathrm{dBnHI} \\
\text { OI } 35 \mathrm{dBnHI}\end{array}$ \\
\hline No3 & 27 & 850 & $7 / 9$ & $\begin{array}{l}\text { VM } 5 \text { días } \\
\text { ATB }\end{array}$ & $\begin{array}{l}50 \mathrm{dBnHI} \text { bilateral } \\
\text { Audífono bilateral }\end{array}$ \\
\hline $\mathrm{N}^{0} 4$ & 27 & 560 & $3 / 7$ & $\begin{array}{l}\text { VM } 12 \text { días } \\
\text { ATB }\end{array}$ & $\begin{array}{c}\text { Sin respuesta a } 90 \mathrm{dBnH} \\
\text { Traslado }\end{array}$ \\
\hline N05 & 33 & 1.440 & $8 / 9$ & - & $80 \mathrm{dBnHL}$ bilateral \\
\hline
\end{tabular}

ATB: Antibióticos (Amikacina, Vancomicina). Bilis: Bilirrubinemia. VM: Ventilación mecánica

viduo, lo que hace necesario la implementación de programas que garanticen esta intervención en salud. Chile es un país en vías de desarrollo, con un gasto en salud limitado. El programa de detección de hipoacusia en el RNPE constituyó un hito muy significativo en nuestra comunidad ya que es el primer esfuerzo hacia la detección universal. Luego de 4 años de ejecución era necesario evaluar sus resultados para sugerir modificaciones y nuevas recomendaciones a las autoridades de salud.

Tanto la tasa de referencia de la etapa de pesquisa $(7,9 \%)$ como la prevalencia de HSN $(1,8 \%)$ están dentro de las cifras esperadas para esta población ${ }^{3-6}$.

Durante la evaluación de la aplicación y ejecución de nuestro programa de detección precoz hubo dos hechos que nos llamaron la atención. En la etapa de pesquisa existieron dos pacientes que fallaron en forma unilateral para luego diagnosticarse HSN bilateral, es decir, dos casos de falsos negativos. La segunda observación que nos parece necesario destacar es la reversibilidad de los resultados del examen de potenciales auditivos evocados, refiriéndonos con esto al hecho de que dos pacientes evolucionaron durante el seguimiento de hipoacusia a normalidad auditiva.

El examen de PEATa es utilizado universalmente en programas de pesquisa precoz de hipoacusia en recién nacidos con factores de riesgo, la presencia de falsos negativos es por lo tanto un hecho relevante, en particular cuando se intenta pesquisar pérdidas auditivas cercanas a los $35 \mathrm{dBnHL}^{7}$. Un factor que podrían explicar estos falsos negativos de los PEATa en casos de hipoacusia leve/moderada se explica por el hecho que la mayoría de los equipos de PEATa presentan el estímulo a $35 \mathrm{dBnHL}$ (click), siendo la referencia para esto un oído adulto ${ }^{8}$ La presión sonora presentada a nivel de la membrana timpánica de un lactante probablemente varía significativamente con respecto a un adulto como consecuencia de la diferencia en volumen del conducto auditivo (ejemplo menor volumen, mayor presión sonora). Debido a esto, un estímulo de $35 \mathrm{dBnHL}$ no necesariamente significa que una pérdida auditiva de $35 \mathrm{~dB}$ (o peor) sea detectada en un niño. En la actualidad no existen estándares (ANSI, ISO) para la calibración de los equipos como los PEATa y de emisiones otoacústicas ${ }^{8}$.

Un segundo factor que podría explicar estos falsos negativos se refiere al valor de la relación señal/ruido de la respuesta detectada. Casi todos los equipos de pesquisa (PEATa) determinan en forma automática la presencia 0 ausencia de respuesta, los procedimientos de medición pueden variar substancialmente de un equipo a otro, los cuales no están estandarizados. Algunos determinan la pre- 
sencia de respuesta comparando los resultados con tablas de valores de normalidad según la edad y sexo. Un número importarte de equipos en la actualidad determinan la presencia de respuesta dependiendo si la medición del PEATa excede un criterio preestablecido (relación señal/ruido) .

La relación señal/ruido utilizado en el PEATa se basa en la magnitud de la respuesta cuando el estímulo está presente (señal) dividido por la magnitud de la respuesta cuando ésta no está presente (ruido). En recién nacidos que presentan respuesta, ésta es mayor que el ruido por lo que la relación señal/ruido presenta un valor mayor que 1,0. En niños donde no se observa respuesta, ésta es aproximadamente similar al ruido por lo que la relación señal/ruido es cercana a 1,0. Inicialmente esta relación se obtenía utilizando la prueba estadística $F$, se utilizaba un punto determinado de la onda del PEAT, esto se denominó Fsp (First single point, algoritmo estadístico), (Don y cols, 1984). En la actualidad los dispositivos de pesquisa con PEATa basan esta relación en múltiples puntos de la onda más que en solo uno en particular, ${ }^{9}$. En la mayoría de los protocolos de evaluación se ha determinado un corte de 3,2 Fsp como criterio PASA. Los dos pacientes portadores de HSN bilateral leve tuvieron falla unilateral/bilateral en la primera etapa de pesquisa. Sin embargo, el oído que pasó la prueba tuvo respuesta de Fsp que varió entre 3,2 y 3,5. Es por esto que hemos iniciado una evaluación prospectiva con especial atención al valor de Fsp como predictor de hipoacusia.

En relación a los pacientes 2 y 3 , en quienes se diagnosticó hipoacusia sensorioneural bilateral, durante el seguimiento evolucionaron hacia la normalidad auditiva. Esto lo denominamos reversibilidad de los resultados, en concordancia con lo reportado en la literatura. Hay dos factores que podrían influir en estos resultados.

Si bien la batería de exámenes aplicada a estos pacientes descartó la presencia de hipoacusia de conducción (timpanograma "A", tono de prueba de $226 \mathrm{~Hz}$ ). El uso de la timpanometría convencional (tono de prueba de $226 \mathrm{~Hz}$ ) no sería lo suficientemente efectiva en la detección de una patología de oído medio en niños menores de 7 meses, es así como existen reportes en donde existe una confirmación de una patología de OM en presencia de timpanogramas " $A$ "11. La pobre sensibilidad de la timpanometría convencional se debería en parte a la falta de desarrollo de la porción ósea del conducto auditivo en recién nacidos. Un aumento en el volumen del conducto mediante presión positiva y su disminución mediante presión negativa puede producir un aumento en la admitancia a medida que se producen cambios de presiones negativas a positivas. Se ha observado que a pesar que el movimiento de la pared del canal no es normal, se puede evidenciar una timpanograma con peak en presencia de efusión ${ }^{12}$. Estos resultados podrían ser debidos a las diferencias en la transmisión acústica del oído medio presentadas entre los recién nacidos y los adultos. Investigaciones indican que el sistema del oído medio en los recién nacidos es "masa dominante", en contraste a un sistema "rigidez dominante" presentado por los adultos. Por esta razón, la timpanometría convencional, que es útil en la evaluación de la función del oído medio en sistemas rigidez dominantes no sería recomendable en los recién nacidos ${ }^{11}$. Según diferentes estudios el uso de un tono de prueba de $1.000 \mathrm{~Hz}$ ha probado ser más sensible en la detección de efusión en los recién nacidos presentando una mejor correlación con la evaluación otorrinolaringológica y con la presencia 0 ausencia de EOAs ${ }^{11,13}$. Por lo tanto, es posible que estos dos casos hayan correspondido a una hipoacusia de conducción no diagnosticada. Apoyado en la literatura existente y considerando lo observado en nuestra casuística sería adecuado la utilización de un tono de prueba de alta frecuencia (ejemplo $1.000 \mathrm{~Hz}$ ) para obtener el timpanograma en recién nacidos de modo de poder establecer la indemnidad del oído medio de una forma más precisa y confiable previa a la consideración de comenzar un proceso de habilitación auditiva.

La segunda opción es un fenómeno que se ha descrito en la literatura como retardo en la maduración de la vía auditiva en recién nacidos de riesgo por alteraciones en la mielinizacion evidenciado por el aumento de las latencias absolutas en particular onda I y $V^{14}$. Este hecho se refleja en la prolongación en las latencias de los potenciales auditivos observados en los recién nacidos en comparación con lactantes mayores y adultos. Sin embargo, resulta difícil comprender cómo el retardo en la mielinizacion podría alterar la aparición de la onda V, alterando los umbrales electrofisiológicos del PEAT, ya que este hecho 
sería aleatorio y no tendría una relación lineal con la edad posgestacional, como sí se ha demostrado para la latencia de ondas ${ }^{15}$.

No fue posible determinar la presencia de falsos positivos ya que el seguimiento audiológico no se realiza en pacientes con examen de pesquisa normal.

\section{CONCLUSIONES}

Los resultados de la ejecución del programa de pesquisa de hipoacusia en esta población muestran indicadores dentro de lo sugerido internacionalmente para estos programas (tasa de referencia, prevalencia hipoacusia de la población estudiada) presentándose resultados similares a lo reportado en la literatura extranjera.

Es importante considerar la posibilidad que el examen de pesquisa tenga falsos negativos, en especial cuando nos enfrentamos a hipoacusias leves a moderadas. Este hecho apoya el seguimiento y evaluación audiológica de los recién nacidos sin importar si éstos refieren uni o bilateralmente.

\section{BIBLIOGRAFÍA}

1. Year 2007 Position Statement: Principles and guidelines for Early Hearing Detection and Intervention Programs. Joint Committee on Infant Hearing. Pediatrics 2007; 120; 898-921.

2. Torrente M, Retamal J, Núñez M. Seguimiento audiológico del recién nacido de muy bajo peso. Rev Otorrinolaringol Cir Cabeza Cuello 2007; 67: 115-21.

3. Yang E, Stuart E, Mencher G, Mencher L, Vincer M. Auditory Brain Stem Responses to Air and Bone Conducted Clicks in the Audiological Assessment at Risk Infants. Ear \& Hearing 1991; 3: 175-82.

4. Cristóbal R, Oghalal JS. Hearing loss in children with very low birth weight: current review of epidemiology and pathophysiology. Arch Dis Child Fetal Neonatal Ed 2008; 93(6): F462-8.

5. Hille ET, van Straaten HI, Verkerk PH. Prevalence and independent risk factors for hearing loss in NICU infants. Acta Paediatr 2007; 96(8): 1155-8.
6. Nazar G, Goycoolea M, Godoy JM, Ried E, Sierra M. Evaluación auditiva neonatal universal: Revisión de 10.000 pacientes estudiados. Rev Otorrinolaringol Cir Cabeza Cuello 2009; 69: 93-102.

7. Durrant JD, Sabo Dl, Delgado Re. Call for calibration standard for newborn screening using auditory brainstem responses. Int $\mathrm{J}$ Audiol 2007; 46(11): 686-91.

8. Johnson JL, White KR, Widen JE, Gravel JS, James M, KenNaLleY T ET AL. A multicenter evaluation of how many infants with permanent hearing loss pass a two-stage otoacoustic emissions/automated auditory brainstem response newborn hearing screening protocol. Pediatrics 2005; 116(3): 66372.

9. Sмітн SD. Performance of a handheld Auditory Brainstem Response Hearing Screener in a wellbaby nursery. [Citado 14 Marzo 2009]. Disponible en: http://www-viasyshealthcare.com/ prod_serv/downloads/SmithPaperABR.pdf

10. SININGER YS, Don M. Effects of click rate and electrode orientation on threshold of the auditory brainstem response. J Speech Hear Res 1989; 32(4): 880-6.

11. Kel J, Allison-Levick J, Dockray J, Harrys R, Kirkegard C Wong J, Maurer M y cols. Highfrequency $(1000 \mathrm{~Hz})$ tympanometry in normal neonates. J Am Acad Audiol 2003; 14(1): 20-8.

12. Margolis RH, Bass-Ringdahl $S$, Hanks WD, Holte $\mathrm{L}$, ZAPALA DA. Tympanometry in newborn infants - $1 \mathrm{kHz}$ norms. J Am Acad Audiol 2003; 14(7): 383-92.

13. Vargas M, Frasson de Azevedo M, Testa JR. Accoustic immitance measures in infants with 226 and 1000 hz probes: correlation with otoacoustic emissions and otoscopy examination. Rev Bras Otorrinolaringol 2009; 75(1): 80-9.

14. Eggermont JJ, Salamy A. Maturational time course for the $A B R$ in preterm and full term infants. Hear Res 1988; 33(1): 35-47.

15. Coenraad S, van Immerzeel T, Hoeve LJ, Goedegebure A. Fitting model of ABR age dependency in a clinical population of normal hearing children. Eur Arch Otorhinolaryngol 2010; 267: 1531-7.

Dirección: TM Oscar Cañete S.

E mail: ocanete@hurtadohosp.cl 